\title{
On the Cooling of Electrons in a Silicon Inversion Layer
}

\author{
O. Prus, M. Reznikov, U. Sivan, \\ Dep. of Physics and Solid State Institute, Technion-IIT, Haifa 32000, Israel \\ and V. Pudalov \\ P. N. Lebedev Physics Institute, 119991 Moscow, Russia
}

\begin{abstract}
Cooling of two-dimensional electrons in silicon-metal-oxide semiconductor field effect transistors is studied experimentally. Cooling to the lattice is found to be more effective than expected from the bulk electron-phonon coupling in silicon. The extracted heat transfer rate to phonons at low temperatures depends cubically on electron temperature, suggesting that another coupling mechanism (such as piezoelectric coupling, absent in bulk silicon) dominates over the deformation potential. According to our findings, at $100 \mathrm{mK}$, the electrons farther than $\sim 100 \mu \mathrm{m}$ from the contacts are mostly cooled by phonons. Using long devices and low excitation voltage we measure electron resistivity down to electron temperature $\sim 100 \mathrm{mK}$ and find that some of the "metallic" curves, reported earlier, turn insulating below $\sim 300 \mathrm{mK}$. This finding renders the definition of the proposed 2D metal-insulator transition questionable. Previous low temperature measurements in silicon devices are analyzed and thumb rules for evaluating their electron temperatures are provided.
\end{abstract}

Since the scaling theory for non-interacting electrons was constructed in the late seventies [1], it was conjectured that a realistic two-dimensional electron system is insulating in the sense that its resistance diverges at low temperatures due to quantum interference and interaction effects. As the temperature is reduced, a logarithmic resistance increase due to weak localization is expected, followed by an exponential resistance divergence once strong localization commences [1]. It was later suggested [2] that strong Coulomb interaction may counteract localization and lead to the existence of a two dimensional metallic state and a metal-insulator transition at zero temperature.

Early experiments on various types of two dimensional electron gases (2DEG) supported localization. For a comprehensive review of early theoretical and experimental results see Refs. [3, 4]. It was therefore unexpected when the existence of a two dimensional metallic phase in high mobility silicon Metal Oxide Semiconductor Field Effect Transistors (MOSFET) was reported in 1994 [5,6]. The ultimate signature of such a metallic phase is resistance saturation to some residual value as the temperature approaches zero [7],ק]. Since $T=0$ is experimentally inaccessible, all claims for a metallic phase rely on extrapolation from finite temperatures. Two traps lurk an experimentalist in this procedure: (a) The assumption that the observed resistance saturation indeed persists to zero temperature may turn wrong. (b) The electron temperature may (and does indeed) depart from the lattice or bath temperature, $T_{b}$, as $T_{b} \rightarrow 0$. The latter point is particularly acute in silicon MOSFETs due to the high intrinsic contact resistance and the weak electron-phonon coupling.

The experiments described here focus on the second point. Using the sample itself as a thermometer we analyze electron cooling as a function of mixing cham- ber temperature and excitation voltage. The main findings are: (a) Electron cooling to the lattice is more effective than expected from the known bulk electronphonon coupling in silicon. Power dissipation is given by $a T^{3}+b T^{5}$ with $a=2.2 \times 10^{-8} \mathrm{~W} / \mathrm{K}^{3} \mathrm{~cm}^{2}$ and $b=5.1 \times 10^{-8} \mathrm{~W} / \mathrm{K}^{5} \mathrm{~cm}^{2}$ and, hence, at temperatures below $\sim 0.6 \mathrm{~K}$, dominated by the $T^{3}$ term [9]. (b) Hot electron diffusion (ED) to the contacts is unimportant in large devices. In short Si MOSFETs, according to our estimates, ED provides cooling or heating depending on the contact resistance. (c) Comparing cooling by phonons with cooling by ED at e.g., $100 \mathrm{mK}$, we find the latter mechanism is typically important only for electrons closer than $\sim 100 \mu \mathrm{m}$ to the contacts. In longer devices, cooling proceeds through phonons. (d) Cooling the electrons down to $100 \mathrm{mK}$ solely by phonons requires an excitation power estimated to be less than $2 \times 10^{-11} \mathrm{~W} / \mathrm{cm}^{2}$. For a $1 \mathrm{~mm}$ square of $2 \mathrm{DEG}$ with $30 \mathrm{~K} \Omega$ resistivity this figure implies an excitation voltage below $80 \mu \mathrm{V}$. A $50 \mu \mathrm{m}$ square with the same resistance requires an excitation voltage below $4 \mu \mathrm{V}$.

Four terminal, lock-in ac measurements at $11 \mathrm{~Hz}$ were carried out on samples similar to those used in Refs. [5, 6]. The samples were $5 \mathrm{~mm}$ long, $0.8 \mathrm{~mm}$ wide Hall bars with $2.5 \mathrm{~mm}$ separation between potential probes. The oxide thickness was $200 \mathrm{~nm}$. Battery powered electrometer preamplifiers were employed to minimize electromagnetic interference and spurious offset bias across the potential probes. With $300 \mathrm{pF}$ cable capacitance, samples up to a few $M \Omega$ total resistance were measured with negligible "out of phase" signal. The excitation voltage across the whole sample was maintained below $100 \mu \mathrm{V}$ to guarantee tolerable heating (this figure is justified below). In order to reduce the contact resistance we applied a larger positive front gate voltage and $-15 \mathrm{~V}$ substrate bias, thus maintaining the same carrier concentration range. The 
substrate bias depletes both the channel and the contacts, but it's effect on the latter is smaller due to heavy doping in the contact regions. This way we were able to reduce the contact resistance below $20 \mathrm{~K} \Omega$ for all "metallic" densities. The substrate bias results in a moderate reduction of the peak mobility from e.g., $3 \times 10^{4} \mathrm{~cm}^{2} / \mathrm{Vs}$ at zero substrate bias to $2.1 \times 10^{4} \mathrm{~cm}^{2} / \mathrm{Vs}$ (both measured at $T=0.3 \mathrm{~K}$ ). The mobility reduction is attributed to enhanced surface scattering due to shift of the $2 \mathrm{DEG}$ towards the $\mathrm{Si}-\mathrm{SiO}_{2}$ interface. The substrate bias was changed in the presence of red LED illumination, which heats the crystal, thus facilitating acceptor recharging in the depletion layer. The high contact resistance problem is further complicated below $1.2 \mathrm{~K}$ by superconductivity of the Al contact metallization. All our "zero magnetic field" measurements were, therefore, carried out in the presence of a $0.01 \mathrm{~T}$ parallel magnetic field which quenched the $\mathrm{Al}$ superconductivity.

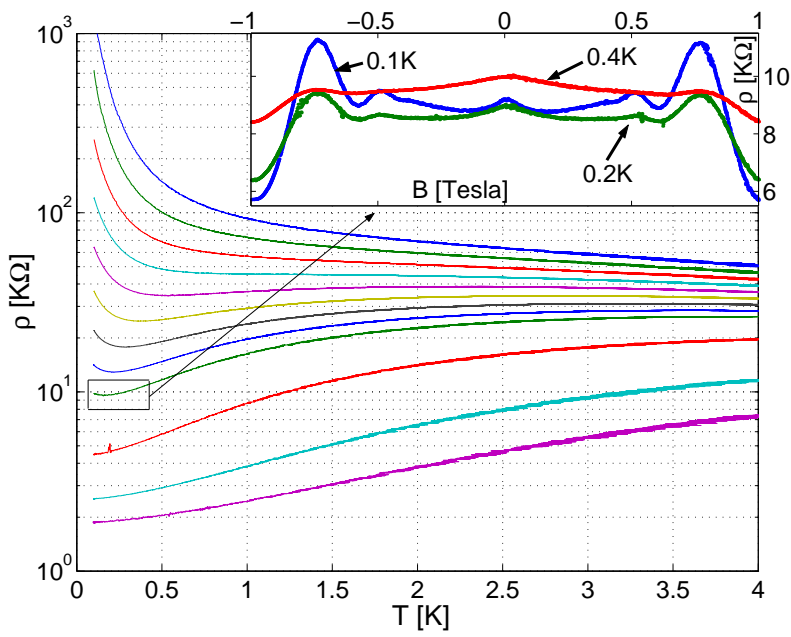

FIG. 1. Temperature dependence of the resistivity. Densities from top to bottom are $n=1.20$ to 1.44 (in 0.03 steps), $1.56,1.8$ and $2.04 \times 10^{11} \mathrm{~cm}^{2} /$ Vs. Inset: Magnetoresistance at $n=1.44 \times 10^{11} \mathrm{~cm}^{2} / \mathrm{Vs}$. The weak localization negative magnetoresistance peak and the onset of Shubnikov - de Haas oscillations are clearly seen. The peak width decreases and the oscillations amplitude increases without saturation as $T_{b}$ decreases to $0.1 \mathrm{~K}$.

The resistivity vs bath temperature for a $2.1 \times$ $10^{4} \mathrm{~cm}^{2} / \mathrm{Vs}$ peak mobility sample at various densities is depicted in Fig. 1. For temperatures above $0.4 \mathrm{~K}$ the traces are similar to those reported in Refs. [5, 6]. At low densities the sample is insulating, while at higher densities the resistance decreases as the temperature is reduced, a behavior previously assigned to a two dimensional metal. For $n=1.29 \times 10^{11} \mathrm{~cm}^{-2}$ the resistance is practically independent of $T$ in a wide temperature range, $0.4 \div 4 \mathrm{~K}$. Such a phenomenon was previously identified as a metal to insulator transition (MIT) [5] [7]. Upon cooling the sample below $0.4 \mathrm{~K}$, we find a novel result; the resistance of some of the "metallic" curves turns up- ward, showing an insulating behavior in all three devices studied by us.

Measurements on a lower mobility sample with $2.5 \times$ $10^{3} \mathrm{~cm}^{2} / \mathrm{Vs}$ peak mobility (Fig. 2) at $0.3 \mathrm{~K}$ yield results similar to those reported earlier [8,10 for the same type of samples. As the temperature decreases, the low mobility sample displays a pronounced insulating behavior at all measured densities, above $6 \times 10^{11} \mathrm{~cm}^{-2}$. A relatively small resistivity drop with cooling is again followed by a resistivity increase at lower temperatures. Such curves have been observed earlier for 2D holes in Si-Ge [11], 2DEG on vicinal Si-surfaces [12], high mobility Si MOSFETs at higher densities, $n>2 \times 10^{12} \mathrm{~cm}^{-2}$ [13, 14, and in $2 \mathrm{D}$ systems on other materials [15,16]. We attribute this resistance upturn to interference and Coulomb interaction effects that grow as the temperature is reduced. The interference is clearly manifested in the weak localization magnetoresistance curves depicted in the inset to Fig. 1.

To gain insight into the electron cooling mechanisms we studied the resistivity dependence upon excitation. We applied a small ac signal superimposed on a dc bias and measured the differential resistivity as a function of this bias. The ac excitation was typically about $100 \mathrm{pA}$, generating less than $65 \mu \mathrm{V}$ ac voltage drop across the $5 \mathrm{~mm}$ sample. The analysis assumes the resistivity depends on the electronic temperature alone and uses the sample resistivity as a thermometer. We chose to use the low mobility sample due to its moderate resistance and monotonic temperature dependence, which facilitate accurate ac measurements.

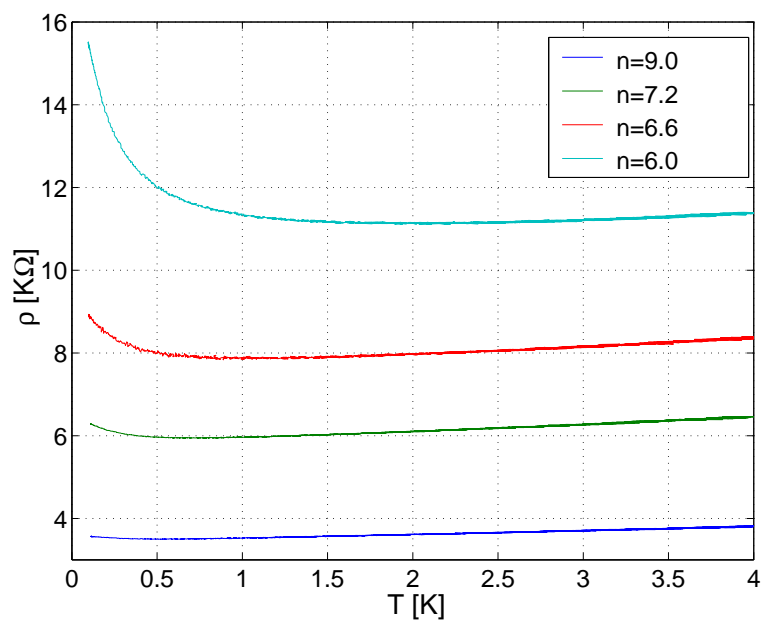

FIG. 2. Resistivity dependence upon temperature for a $2.5 \times 10^{3} \mathrm{~cm}^{2} / \mathrm{Vs}$ peak mobility sample. Densities given in $10^{11} \mathrm{~cm}^{2} / \mathrm{Vs}$ units.

Inset (a) to Fig. 3 depicts the channel resistivity versus dc current at various bath temperatures. The overall dissipated power per unit area of the 2DEG consists of Joule heating, $P_{d}=J^{2} \times \rho$, by the driven current and heating by spurious excitations, $P_{0}$, due to e.g., absorp- 
tion of electromagnetic radiation. The heat is dumped to phonons and to the contacts via electron diffusion. We ignore for the moment electron diffusion - an assumption to be later justified for our samples. In the general case, electron-phonon interaction may be due to both piezoelectric and deformation potential coupling with their characteristic $T^{3}$ and $T^{5}$ dependencies 17, respectively. Similar functional form was recently found for holes in SiGe heterostructures [18]. We therefore characterize the electron phonon interaction by the coupling parameters $a$ and $b$.

$$
P_{d}+P_{0}=a\left(T_{e l}^{3}-T_{b}^{3}\right)+b\left(T_{e l}^{5}-T_{b}^{5}\right)
$$

We solve Eq. 1 to find the electron temperature, $T_{e l}$, and fit $a$ and $b$ in such a way that all $R\left(T_{e l}\right)$ data in inset (a) to Fig. 3 collapse to a single curve. Figure 3 shows the best fit obtained with $a=2.2 \times 10^{-8} \mathrm{~W} / \mathrm{K}^{3} \mathrm{~cm}^{2}$ and $b=5.1 \times 10^{-8} \mathrm{~W} / \mathrm{K}^{5} \mathrm{~cm}^{2}$. $P_{0}$ cannot be determined in this scaling procedure. The piezoelectric contribution is, hence, found to dominate electron - phonon coupling at all temperatures below $0.6 \mathrm{~K}$. This effect is unexpected since a Si lattice possesses inversion symmetry and, hence, should display deformation potential coupling only. We believe the piezoelectric contribution, found in our experiment, originates from the $\mathrm{Si}-\mathrm{SiO}_{2}$ interface which lacks inversion symmetry.

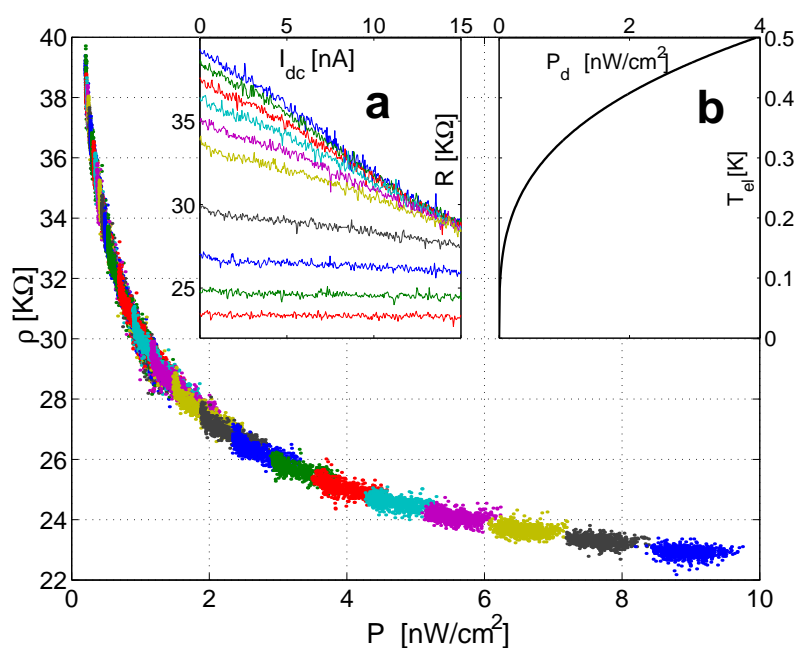

FIG. 3. Channel resistance scaling for the low mobility sample. The abscissa depicts $P=P_{d}+a T^{3}+b T^{5}$. The fitting parameters are: $a=2.2 \times 10^{-8} \mathrm{~W} / \mathrm{K}^{3} \mathrm{~cm}^{2}$ and $b=5.1 \times 10^{-8} \mathrm{~W} / \mathrm{K}^{5} \mathrm{~cm}^{2}$. Colors correspond to the same curves as in inset a. Insets: a) resistivity versus dc current at different base temperatures: 90, 120, 150, 180, $210,240,330,420,510,600 \mathrm{mK}$. b) the electronic temperature calculated using eq. 1 as a function of dissipated power for $T_{b}=0, P_{0}=0$.

Heat transfer due to ED can be incorporated into the model using the Wiedemann-Franz law [19]. The power balance reads

$$
a\left(T_{e l}^{3}-T_{b}^{3}\right)+b\left(T_{e l}^{5}-T_{b}^{5}\right)=
$$

$$
P_{d}+P_{0}+\frac{1}{6}\left(\frac{\pi k_{B}}{e}\right)^{2} \sigma \frac{\partial^{2} T^{2}}{\partial x^{2}}
$$

where $\sigma$ is the sample conductivity. At temperatures $T_{e l} \ll 0.6 \mathrm{~K}$ the deformation potential contribution can be ignored. Defining $T_{0}^{3}=T_{b}^{3}+\left(P_{d}+P_{0}\right) / a$ and

$$
\lambda=\frac{\pi k_{B}}{3 e} \sqrt{\sigma / a T_{0}},
$$

Eq. 2 takes the dimensionless form

$$
\Theta^{3}-1=\frac{3}{2} \frac{\partial^{2} \Theta^{2}}{\partial \chi^{2}}
$$

where $\Theta \equiv T_{e l} / T_{0}$ and $\chi \equiv x / \lambda . \quad \lambda$ sets the characteristic length scale on which the $T_{e l}$ approaches $T_{0}$. The only temperature scale in the problem is $T_{0}$, the electron temperature far away from the contacts, where cooling is dominated by phonons. Equation 4 can be integrated exactly. Away from the contacts, where $\Theta \approx 1, \Theta \approx$ $1+\exp (-\chi)$. For $\left.\Theta \gg 1, \Theta(\chi) \approx \Theta_{c} /\left(1+\sqrt{\left(\Theta_{c} / 30\right.}\right) \chi\right)^{2}$, where $\Theta_{c}$ is the normalized electron temperature near the contact.

For $T_{0}=100 \mathrm{mK}$ and $\sigma=3.3 \times 10^{-5} \mathrm{Ohm}^{-1}$ (typical critical region conductance), $\lambda \approx 110 \mu \mathrm{m}$. For our $5 \mathrm{~mm}$ long samples, ED is, hence, negligible and Eq. 1 can be safely used to estimate $T_{0}$. The resulting electron temperature as a function of dissipated power, is plotted in inset (b) to Fig. 3. Assuming that the electron-phonon coupling does not depend significantly on impurity scattering we use the same parameters to analyze the high mobility sample data. Equation 11 shows that the overheating due to the measurement current is negligible and does not exceed $1 \mathrm{mK}$ for a $50 \mathrm{mK}$ base temperature. We have used the magnetoresistance curves shown in the inset to Fig. 1 to estimate the lowest sample temperature by fitting the curves to weak localization theory. The extracted dephasing rate scales linearly with temperature, in agreement with theory 何, down to the lowest analyzed temperature, $T_{b}=100 \mathrm{mK}$. Although we use weak localization theory for resistivities beyond its applicability, the linear dependence of the dephasing rate upon bath temperature indicates that electrons cool down to $T_{e l} \sim 100 \mathrm{mK}$.

We turn now to investigate the implications of our results to previous measurements. Surprisingly, there are only a few published experiments below $300 \mathrm{mK}$. Those experiments were carried out on two types of samples. The first type, in which the MIT was originally observed [5.6], is a conventional MOSFET that, in the lack of back gating, suffers from high contact resistance, especially at low electron densities and temperatures. These samples are several $\mathrm{mm}$ long, and heat transfer by electron diffusion to the contacts can be safely neglected. In the interesting regime, where the resistance drop is significant, the contact resistance is in the $\mathrm{M} \Omega$ range. Such a 
high contact resistance dictates either dc or very low frequency measurements which require, due to $1 / f$ noise in the amplifiers and the sample, substantial excitation currents. Overheating is evident in both $R$ vs $T$ and $\ln R$ vs $1 / T$ dependencies (see e.g., Figs. 1 and 5 in [6] and Fig. 3 of [20]), even in the insulating regime where it should be less significant. In previous experiments [21,22, where $T_{e l}$ was measured in the "metallic" regime, it was never lower than $\approx 200 \div 300 \mathrm{mK}$.

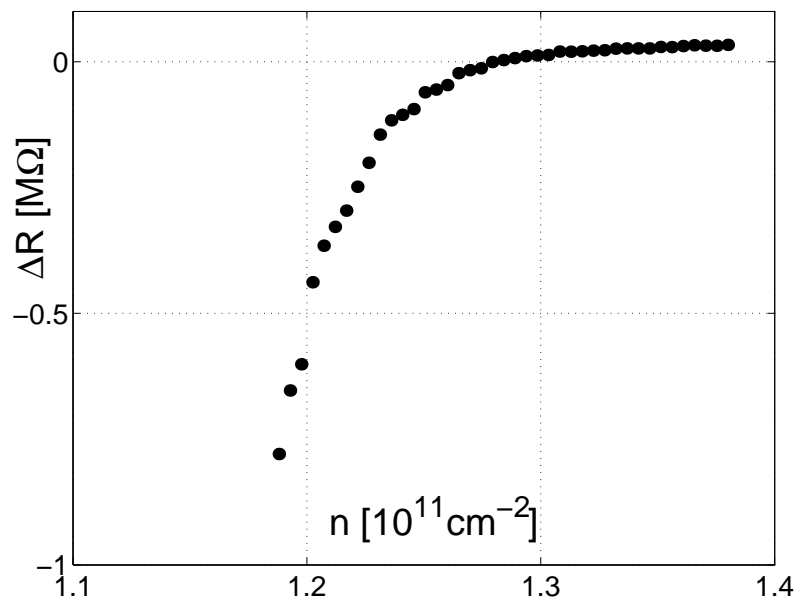

FIG. 4. The difference between the two terminal resistance, $R_{2}$, and the ideal resistance, $\tilde{R}_{2}=\rho \times L / W$, corresponding to a uniform sample temperature and zero contact resistance. $T_{b}=80 \mathrm{mK}$ and the excitation voltage $\leq 65 \mu \mathrm{V}$. Note the difference changes sign at approximately the MIT density.

In another type of samples 23,24, the gates, adjacent to the contacts, are separated from the gate over the central part of the sample by $\approx 50 \mathrm{~nm}$ gaps. This arrangement facilitates a higher carrier concentration in the contact regions and, hence, a reduced contact resistance. In contrast to the millimeters long samples, employed here and in Refs. [5,60|, the effective sample length between the gaps in Ref. [24] is only $120 \mu \mathrm{m}$. Substituting $\lambda=60 \mu \mathrm{m}$ into Eq. 3 and solving for $T_{0}$ we find that cooling is mostly provided by phonons for all temperatures above $\sim 340 \mathrm{mK}$. Since cooling by phonons is suppressed only slightly faster than by $\mathrm{ED}\left(T^{3}\right.$ compared with $\left.T^{2}\right)$, phonons, neglected in the analysis of Ref. 224 done in Ref. [8], are important also at lower temperatures. Electron diffusion from the contacts may either cool or warm electrons in the central part of the sample, depending on the voltage drop, $U_{c}$, across the $\approx 50 \mathrm{~nm}$ gap. For a given electron temperature near the contact, $T_{c}$, one can estimate the voltage drop, $U_{c}$, across the gap resistance, $\rho_{c}$, for which the net heat flow between the contact and the sample vanishes. Using Wiedemann-Franz law, the heat flow balance reads

$$
U_{c}^{2} / 2 \rho_{c}=\frac{\pi^{2}}{6 \rho_{c}}\left(\frac{k_{b}}{e}\right)^{2}\left(T_{c}^{2}-T_{b}^{2}\right),
$$

For $T_{b}=0$ one obtains $U_{c} / T_{c}=\left(\pi k_{b} / \sqrt{3} e\right) \sim$ $156 \mu \mathrm{V} / \mathrm{K}$, independent of $\rho_{c}$. For lower (higher) voltages across $\rho_{c}$, electrons are cooled (warmed) by the contacts. For $100 \mathrm{mK}$, the bias at which the contacts neither heat nor cool the sample equals $15.6 \mu \mathrm{V}$. At that bias sample cooling is provided by phonons. Using Eq. 1 we find that $100 \mathrm{mK}$ electron temperature in a $30 \mathrm{k} \Omega$ per square sample requires less than $\sim 4 \mu \mathrm{V}$ voltage drop per $50 \mu \mathrm{m}$ square, which for the sample in Ref. [24], translates into $10 \mu \mathrm{V}$ between the gate gaps. A $100 \mathrm{mK}$ electron temperature throughout the sample thus requires less than $\sim 40 \mu \mathrm{V}(50 \mathrm{mK}$ requires $<20 \mu \mathrm{V}$ ) bias across the whole sample (neglecting the voltage drop across the high density contacts). Electron overheating in the contact regions occurs in our samples, as illustrated in Fig. 4. The contact regions are always more resistive than the rest of the sample in the "metallic" regime, $n>1.29 \times 10^{11} \mathrm{~cm}^{-2}$, and less resistive in the insulating one, $n<1.29 \times 10^{11} \mathrm{~cm}^{-2}$, indicating they are warmer than the rest of the sample.

In summary, we found that the cooling of electrons by phonons in Si MOSFETs is substantially more effective than that expected from the bulk electron-phonon coupling. Long samples are cooled by phonons. Short samples are susceptible to heat transfer by ED from the contacts. Low temperature measurements in short samples require low excitations. Using $5 \mathrm{~mm}$ long devices, where electron heating in the contacts can be neglected, we were able to measure electron resistivity down to $\sim 100 \mathrm{mK}$ temperatures. We found that $\rho(T)$-curves in the well pronounced "metallic" regime, corresponding to $(1.3-1.44) \times 10^{11} \mathrm{~cm}^{-2}$ densities and a three fold resistance reduction with cooling, turn insulating below a certain temperature. That temperature is $\sim 300 \mathrm{mK}$ at $\rho \sim h / e^{2}$ and decreases as the density is increased and $\rho$ decreases. Our finding renders the definition the $2 \mathrm{D}$ metallic phase and the metal-insulator phase transition questionable. Further work is needed to determine the physics behind the observed resistance upturn.

We have benefited greatly from valuable discussions with Y. Yaish, M. Gershenson, A. Finkelstein, A. Stern, and T. M. Klapwijk. This work was supported by the Israeli National Science foundation, the DIP foundation, INTAS, NSF, NATO Scientific Program, RFBR, and the Russian programs: "Physics of Nanostructures", "Statistical Physics", "Integration", and "The State Support of Leading Scientific Schools".

[1] E. A. Abrahams, P. W. Anderson, D. C. Licciardello, and T. V. Ramakrishnan, Phys. Rev. Lett. 42673 (1979).

[2] A. M. Finkelstein, JETP 84, 168 (1983). Z. Phys. B 56, 
189 (1984)

[3] P. A. Lee, T. V. Ramakrishnan, Rev. Mod. Phys. 57, 287 (1985).

[4] B. L. Altshuler and A. G. Aronov, in Electron-electron interactions in disordered systems, ed. by A. L. Efros and M. Pollak , p. 1 (Elsevier, Amsterdam, 1985).

[5] S. V. Kravchenko, G. V. Kravchenko, J. E. Furneaux, V. M. Pudalov, M. D'Iorio, Phys. Rev. B 50, 8039 (1994), cond-mat/9405082.

[6] S. V. Kravchenko, W. E. Mason, G. E. Bowker, J. E. Furneaux, V. M. Pudalov, M. D'Iorio, Phys. Rev. B 51, 7038 (1995), cond-mat/9412103.

[7] E. Abrahams, S. V. Kravchenko, M. P. Sarachik, Rev. Mod. Phys. 73, 251 (2001), cond-mat/0006055.

[8] B. L. Altshuler, D. L. Maslov, and V. M. Pudalov, Physica E, 9, 209 (2001), cond-mat/0003032.

[9] This form is equivalent to the empirical law, $\sim T^{3.3}$ over the range $T=0.2 \div 4 \mathrm{~K}$.

[10] V. M. Pudalov, G. Brunthaler, A. Prinz, G. Bauer, Physica E 3, 79 (1998).

[11] V. Senz, T. Heinzel, T. Ihn, K. Ensslin, G. Dehlinger, D. Grützmacher, and U. Gennsher, Phys.Rev.B 61, 5082 (2000), cond-mat/9910228.

[12] S. S. Safonov, S. H. Roshko, A. K. Savchenko, A. G. Pogosov, Z. D. Kvon, Phys. Rev. Lett. 86, 272 (2001), cond-mat/0003060.

[13] V. M. Pudalov, G. Brunthaler, A. Prinz, and G. Bauer, Phys. Rev. B 60, 2154 (1999), cond-mat/9801077.

[14] B. L. Altshuler, G. W. Martin, D. L. Maslov, V. M.
Pudalov, A. Prinz, G. Brunthaler, G. Bauer, condmat/0008005.

[15] Y. Y. Proskuryakov, A. K. Savchenko, S. S. Safonov, M. Pepper, M. Y. Simmons, D. A. Ritchie, Phys. Rev. Lett. 86, 4895 (2001), cond-mat/0101195.

[16] E. Ribeiro, R. Jaäggi, T. Heinzel, K. Ensslin, G. Medeiros-Ribeiro, and P. M. Petroff, Phys. Rev. Lett., 82, 996 (1999), cond-mat/9808276.

[17] V. Karpus, Semic. Sci. Technol. 5, 691 (1990).

[18] R. Leturcq, D. L'Hote, R. Tourbot, V. Senz, U. Gennser, T. Ihn, K. Ensslin, G. Dehlinger, D. Gr?tzmacher, condmat/0107457.

[19] Wiedemann-Franz law remains valid in Fermi-liquid in presence of interactions (see M. Reizer, Phys. Rev. B 44, 12701 (1991)).

[20] M. D'Iorio, V. M. Pudalov, and S. G. Semenchinsky, Phys. Rev. B 46, 15992 (1992).

[21] G. Brunthaler, A. Prinz, G. Bauer, and V. M. Pudalov, cond-mat/0007230. PRL (2001), in press.

[22] V. M. Pudalov, M. Gershenson, H. Kojima, N. Butch, E. M. Dizhur, G. Brunthaler, A. Prinz, G. Bauer, condmat/0105081. M. Gershenson et al., in the EP2DS-14 workbook, Prague (2001).

[23] R. Heemskerk, "Transport at Low Electron Densitity in the Two-Dimensional Electron Gas of Silicon MOSFETs" PhD Thesis, Rijksuniversiteit Groningen (1998), adviser: T. M. Klapwijk.

[24] S. V. Kravchenko and T. M. Klapwijk, Phys. Rev. Lett. 84, 2909 (2000), cond-mat/9909458. 